\section{Diagnosing poisoning in practice: interactive case discussion}

\section{Nick Carmichael}

In this case-based interactive session a number of common canine toxicological presentations will be discussed.

Following oral ingestion, vitamin $\mathrm{K}$ antagonist rodenticides have a short circulating half-life in blood and are rapidly cleared by the liver. These agents, however, exert significant and prolonged inhibition of the enzyme system involved in the production of active vitamin K1 within the liver for days to weeks. Clinicians may therefore face the paradoxical situation of a patient with signs and laboratory results compatible with vitamin $\mathrm{K}$ antagonist toxicity in which the agents themselves cannot be identified in blood. Blood analysis for active vitamin K1 and particularly vitamin K1 2,3 epoxide which accumulates and persists in blood in the presence of these agents can be useful to confirm toxicity.

Blue pellets containing metaldehyde are commonly available but are not the only toxic agent found in this form which is capable of causing seizures in dogs. The case presented demonstrates the clinical features including the so-called SLUDGE signs which assist differentiation of intoxication with carbamate/organophosphate from metaldehyde and the utility of postmortem toxicological analysis in some cases to assist in confirming the diagnosis.

Neuromycotoxicosis is associated with ingestion of mouldy foods containing toxins derived from specific Penicillium species. The clinical appearance (tremors and convulsions) can be very similar to intoxication with bromethalin. Identification of these agents requires the use of specific analytical techniques and they will not be detected on routine toxicological analysis. The presented case illustrates both the importance of developing an analytical plan for toxic agents likely to be involved based on the clinical findings and the importance of appropriate sample selection.

\section{KEY LEARNING OBJECTIVES}

- Understand the rapid clearance of vitamin $\mathrm{K}$ antagonist rodenticides from blood and the consequent diagnostic utility of vitamin K1 2,3 epoxide assay in supporting toxicity with these rodenticides

- Have an awareness of the range of blue-coloured toxic materials available and the use of clinical signs and toxicological analysis to assist differentiation of these agents and their appropriate treatment

- Understand the importance of appropriate sampling and toxicological analytical method selection to assist differentiation of potential causes of tremors and convulsions in dogs

\section{MULTIPLE CHOICE QUESTIONS}

1. Gas chomatography-mass spectrometry analysis can identify many thousands of compounds, including many common toxins, in a single analysis. Which common toxin CANNOT be reliably detected by this method?
(A) Theobromine (chocolate)
(B) Ibruprofen
(C) Penitrem $\mathrm{A}$
(D) Paracetamol

2. Why is measurement of vitamin $K 12,3$ epoxide useful in suspected vitamin $\mathrm{K}$ antagonist rodenticide poisoning?
(A) It is a breakdown metabolite of these agents
(B) It remains increased in blood for weeks following vitamin $\mathrm{K}$ antagonist exposure
(C) It is proportionate to the amount of active vitamin $\mathrm{K}$ in blood
(D) It is a prognostic indicator of recovery from intoxication

3. So-called SLUDGE signs are associated with organophosphate/carbamate poisoning. They arise from over stimulation of which part of the nervous system?
(A) Sympathetic nervous system
(B) Reticular activating system (RAS)
(C) Vestibular system
(D) Parasympathetic nervous system

\section{How lab work helps me unravel the cause of a body cavity effusion}

\section{Elizabeth Villiers}

Assessing the nucleated and red cell counts, protein with or without other biochemical tests and cytology of an effusion helps identify its causes. Protein is estimated with a refractometer or measured on the biochemistry analyser. If feline infectious peritonitis (FIP) is suspected, the albumin:globuin ratio should be measured $\quad<0.8$ in FIP). For cytology a direct and sediment smear, or cytospin smear are made.

In septic peritonitis the effusion is turbid with a nucleated cell count of $>13 \times 10^{9} / /$ comprising predominantly degenerate neutrophils, some of which contain bacteria. The glucose concentration is typically low and the lactate concentration high, although these can also be seen in sterile inflammation. In bile peritonitis, macrophages containing green-brown bile pigment are present. The fluid bilirubin is higher than the serum bilirubin. Peritonitis caused by pancreatitis leads to a sterile mainly neutrophilic exudate with macrophages which may contain 


\section{Clinical pathology}

phagocytosed fat vacuoles. The fluid lipase concentration is higher than the serum lipase concentration.

Uroabdomen leads to an azotaemia with hyperkalaemia and often hyponatraemia. It typically leads to a transudate, or with longer-standing uroabdomen, inflammation may develop. The fluid creatinine:serum creatinine ratio is $>2$ and the fluid potassium:serum potassium is $>1.4$.

Chylous effusions have a triglyceride concentration of $>1.4 \mathrm{mmol} / \mathrm{l}$ and a cholesterol:triglyceride ratio of $<1$ (ratio calculated after converting to $\mathrm{mg} / \mathrm{dl}$ ). They form due to rupture or obstruction of, or increased pressure on, lymphatics or may be idiopathic. Cats with cardiac disease may have lymphocyte-rich effusions without elevated triglyceride.

Transudates are classified as protein-poor (protein $<20 \mathrm{~g} / \mathrm{l}$ ), which usually form due to hypoalbuminaemia; and protein-rich (protein $>20 \mathrm{~g} / \mathrm{l}$ ), which form due to increased hydrostatic pressure in veins or lymphatics due to cardiac disease, neoplasia, thrombosis or liver disease. They have low numbers of nucleated cells $\left(<5 \times 10^{9} /\right.$ l) with a mix of macrophages, neutrophils, mesothelial cells and lymphocytes.

\section{KEY LEARNING OBJECTIVES}

- Outline the approach to evaluation of body cavity effusions in the practice laboratory

- Be able to distinguish transudates and exudates and identify their underlying cause

- Identify patterns in haematology and biochemistry accompanying effusions

\section{MULTIPLE CHOICE QUESTIONS}

1. A 12-year-old cat has an amber slightly cloudy pleural effusion.

Protein $35 \mathrm{~g} / \mathrm{l}$
Albumin $18 \mathrm{~g} / \mathrm{l}$

Globulin $17 \mathrm{~g} / \mathrm{l}$

Nucleated cells $3.8 \times 10^{9} / \mathrm{l}$

$\mathrm{HCT}<1$

Cytology: $80 \%$ small lymphocytes, $15 \%$ macrophages, $5 \%$ neutrophils

What is the most likely cause of this effusion?
(A) FIP
(B) High-grade lymphoma
(C) Cardiac disease
(D) Ruptured thoracic duct

2. Which of the following would be expected in septic peritonitis:
A Elevated serum protein and albumin
B Effusion nucleated cell count $>13 \times 10^{9} / \mathrm{l}$
C Predominance of macrophages in the abdominal effusion
D Bacteria within neutrophils
E Bacteria within macrophages
(A) A, B, D
(B) $B, E$
(C) $A, B, D$
(D) $B, D$

3. A 7-year-old Golden Retriever has a cranial mediastinal mass and a pleural effusion with a nucleated cell count of $28 \times 10^{9} /$, consisting of large lymphoid cells with a large nucleus containing stippled chromatin and two to three small nucleoli. Which of the following is causing this effusion?
(A) Thymoma
(B) Lymphoma
(C) Cardiac disease
(D) Ruptured thoracic duct

\section{Nuts and bolts of culture and susceptibility testing}

\section{Elizabeth Villiers}

In order to obtain accurate results in culture and susceptibility testing it is important to follow strict guidelines set out by regulatory bodies such as the Clinical and Laboratory Standards Instititute (CLSI) and the European Committee on Antimicrobial Susceptibility Testing (EUCAST).

Swabs are plated out on to various agar plates using a streak technique, which, after incubation, produce individual colonies which are scooped off for analysis. A Gram stain and basic biochemical tests such as oxidase and catalase tests are performed. These preliminary results, as well as the appearance of the colonies, will determine selection of subsequent biochemical tests which are performed using colorimetic strips or with automated systems such as the Vitek analsyer. Results are compared to a database and algorithms used to generate the identification. Alternatively bacteria can be identified using matrix-assisted laser desorption/ionization time of flight analysis (MALDI-TOF).

Gram-negative rods are divided into those that ferment glucose (Pasteurella and Enterobacteriaceae) and non-fermenters. Enterobacteriaceae include Escherichia coli, Klebsiella, Proteus, Enterobacter, Citrobacter and Serratia. These organisms commonly cause urinary tract, wound, soft tissue, body cavity and ear infections. Non-fermenters include Pseudomonas aeruginosa (a common cause of ear, eye and respiratory infections); Acinetobacter and Burkholderia (which can cause nosocomial infections). Non-fermenters have extensive intrinsic resistance to multiple antibiotics. Gram-negative cocci include Neisseria (which can cause oral infections) and Moraxella, which cause respiratory infections. 